\title{
The thermodynamics of heavy light hadrons at freezeout
}

\author{
Sayantan Sharma (for the Bielefeld-BNL-CCNU Collaboration) ${ }^{1}$ \\ Fakultät für Physik, Universität Bielefeld, D-33615 Bielefeld, Germany
}

\begin{abstract}
In the discussion of hadronization at or close to the freeze-out curve statistical (hadron resonance gas) models play an important role. In particular, in the charmonium sector, regeneration models are considered which rely on the fact that charmonium states can form again already at temperatures well above the QCD transition or hadronization temperature. An important ingredient in these considerations is the regeneration or hadronization of open charm states. In this talk we report on a lattice QCD analysis of correlations of open strange and charm with other conserved quantum numbers like the net baryon number and electric charge. We analyze the temperature range in which an uncorrelated hadron resonance gas (HRG) provides an adequate description of such correlations. This limits the range of validity of HRG based thermodynamics in open flavor channels and provides an estimate for the melting temperature of heavy-light hadrons.
\end{abstract}

Keywords: charmed baryons, open charm mesons, hadron resonance gas, charm quark susceptibilities, QCD thermodynamics, lattice gauge theory

\section{Introduction}

The strongly interacting matter described with Quantum Chromodynamics (QCD) shows many interesting features like deconfinement and chiral symmetry breaking. For QCD with light quark flavours in the fundamental representation, the chiral symmetry restoration and deconfinement of the light quark degrees of freedom occur at the same temperature which is known as the chiral crossover temperature $T_{c}$. It is interesting to understand whether the same phenomenon occurs for the heavier strange and charm quarks. It is known that the bound states of charm quarks and its anti-particle like $J / \psi, \eta_{c}$ deconfine at temperatures about $1.5 T_{c}[1,2]$ and serve as a probe of the presence of a quark gluon plasma [3]. Recently it has been shown from first principles lattice study that the open strange hadrons melt at $T_{c}$ [4], confirmed through another independent analysis [5]. The charm quark mass is an order of magnitude larger than the strange mass and it is important to understand when the open charm hadron states do melt. Apart from being a problem of fundamental importance, it has phenomenological implications too. At LHC energies, it is expected that the charmonium states would be regenerated at the freezeout temperatures [6]. In the statistical model of hadronization inspired by the hadron resonance gas (HRG) model, the open charm hadrons would contribute to the regeneration of the charmonium states through feed down corrections. For these studies, it is important to know the hadron content at the freezeout. In this contribution we address two issues pertaining to the hadrons made of light and heavy quark flavours especially in the charm sector. From first principles lattice study we estimate the melting

\footnotetext{
Email address: sayantan@physik.uni-bielefeld.de (Sayantan Sharma (for the Bielefeld-BNL-CCNU Collaboration))

${ }^{1}$ A. Bazavov, H.-T. Ding, P. Hegde, O. Kaczmarek, F. Karsch, E. Laermann, Y. Maezawa, S. Mukherjee, H. Ohno, P. Petreczky, C. Schmidt, S. Sharma, W. Soeldner and M. Wagner
} 

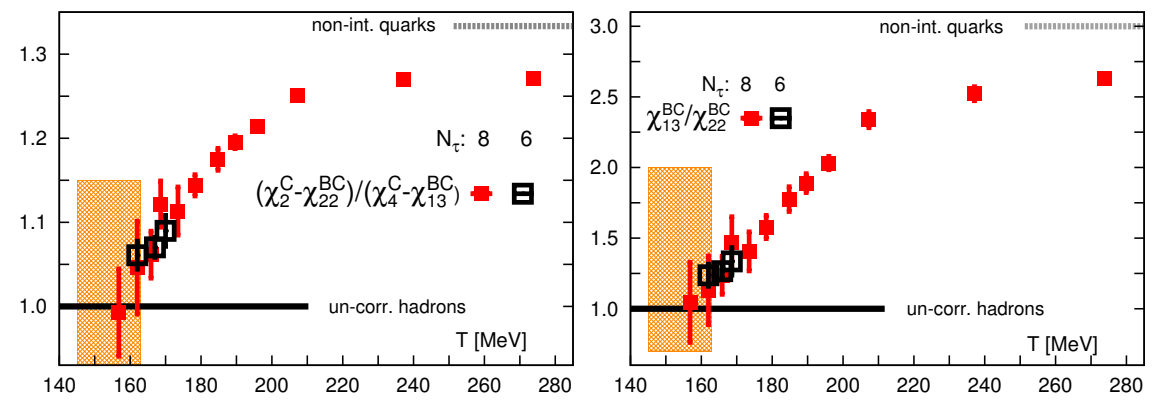

Figure 1. The ratios of the partial pressure of the open charm mesons (left panel) and baryons (right panel) are shown as function of temperature for two different lattice sizes with $N_{\tau}=6,8$. The yellow band represents the crossover region.

temperature for the open charm mesons and baryons independently of the hadron content. We then proceed to show that QCD thermodynamics at the chiral crossover transition provides hints about the existence of many more charmed baryons which are yet to be detected in the experiments.

\section{Melting of open charm hadrons}

The total pressure of a thermal ensemble of a non-interacting gas of open charm mesons and baryons and resonances at finite chemical potential $\hat{\mu}_{X}=\mu_{X} / T, X \equiv B, C$ is given as,

$$
P\left(\hat{\mu}_{C}, \hat{\mu}_{B}\right)=P_{M} \cosh \left(\hat{\mu}_{C}\right)+P_{B, C=1} \cosh \left(\hat{\mu}_{B}+\hat{\mu}_{C}\right)+P_{B, C=2} \cosh \left(\hat{\mu}_{B}+2 \hat{\mu}_{C}\right)+P_{B, C=3} \cosh \left(\hat{\mu}_{B}+3 \hat{\mu}_{C}\right) .
$$

Motivated from the analysis developed in Ref. [4], we can rewrite the partial pressures in the baryon and the meson sectors in terms of susceptibilities at $\hat{\mu}_{X}=0$. The derivatives of $P\left(\hat{\mu}_{C}, \hat{\mu}_{B}\right)$ with respect to $\mu_{X}, \chi_{k l}^{B C}=\left.\frac{\partial^{(k+l)}\left[P\left(\hat{\mu}_{B}, \hat{\mu}_{C}\right) / T^{4}\right]}{\partial \hat{\mu}_{B}^{k} \partial \hat{\mu}_{C}^{l}}\right|_{\vec{\mu}=0}$, yield different susceptibilities which denote the fluctuations of $B, C$ and their correlations. In a non-interacting gas of charmed hadrons, these quantities can be written as,

$$
\chi_{n}^{C}(\hat{\mu}=0)=P_{M}+1^{n} P_{B, C=1}+2^{n} P_{B, C=2}+3^{n} P_{B, C=3}, \chi_{k l}^{B C}(\hat{\mu}=0)=1^{l} P_{B, C=1}+2^{l} P_{B, C=2}+3^{l} P_{B, C=3} .
$$

Inverting these relations would give us the partial pressures in the meson and baryon sectors. We calculate correlations between the conserved quantum numbers, net baryon number, charm and their fluctuations upto fourth order at $\mu_{X}=0$ on the lattice on $2+1$ flavour Highly Improved Staggered Quark (HISQ) configurations with quenched charm quark. The strange quark mass is set to its physical value and the light quark mass is chosen such that $m_{\pi}=160 \mathrm{MeV}$ in the continuum. The charm quark mass is fixed by setting the spin-averaged charmonium mass [7], $\frac{1}{4}\left(m_{\eta_{c}}+3 m_{J / \psi}\right)$ to its physical value. Two different lattices with spatial size $N_{s}=32,24$ and temporal extent $N_{\tau}=8,6$ respectively are used for this study. The crossover temperature is $T_{c}=154(9) \mathrm{MeV}$ [8] in the continuum limit for physical quarks. We analyzed susceptibilities in the temperature range 156-330 MeV. To control systematic errors, 6000 stochastic sources were employed to compute the fluctuations in the charm sector and 1500 for the light sector. About 16000 configurations were considered at the lowest temperature and 1600-6000 configurations at high temperatures to control the statistical errors. The computational details are outlined in [9].

The $P_{M}$ can be expressed as two equivalent combinations of the susceptibilities, $P_{M}=\chi_{2}^{C}-\chi_{22}^{B C}=\chi_{4}^{C}-\chi_{13}^{B C}$. The ratio of the two independent measures of $P_{M}$ would be unity in the hadron phase and deviation from unity would be a signal of the melting of the open charm mesons. In the baryon sector, $C=2,3$ baryons are substantially heavier than the $C=1$ baryons and their contribution to the total pressure has been shown to be negligibly small compared to $P_{B, C=1}$ [9]. It follows that $P_{B=1} \simeq \chi_{m n}^{B C}, m, n>0$ and $m+n=$ even. Ratios like $\chi_{13}^{B C} / \chi_{22}^{B C}$ would be unity in a phase of charmed baryons. The ratios in the charmed meson and baryon sectors are shown in Fig. 1. It is evident that both the open charm mesons and baryons melt at the crossover. These ratios are independent of the details of the hadron spectrum and have relatively small lattice cut-off effects evident from the $N_{\tau}$ independence of our results. 


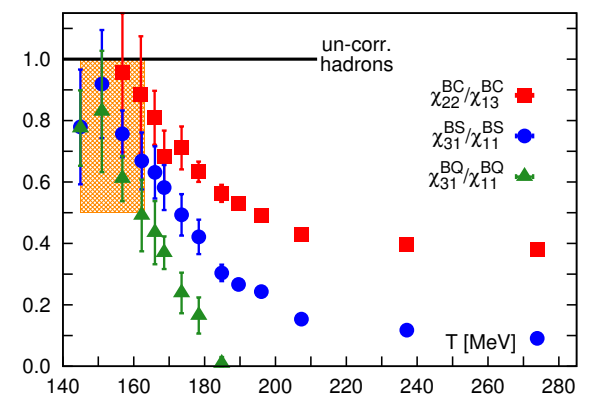

Figure 2. The ratios of susceptibilities which probe the charm (BC) baryon, strange baryon (BS) and dominantly the baryons with light quark flavours (BQ) all show a departure from unity at the crossover region (shaded yellow).

The behaviour of the light, strange and the charm baryons near the crossover are summarized in Fig. 2 through the ratios of fluctuations which are chosen such that these are unity in the hadron phase. The lattice data in these sectors supports that deconfinement of the open heavy hadrons occur at the crossover transition region evident from the rapid departure of unity beyond $\sim 160 \mathrm{MeV}$.

\section{Evidence of additional charm hadrons from QCD thermodynamics}

Our analysis allows us to disentangle the partial pressures of the meson and baryon sectors with different quantum numbers and understand the composition of these individual sectors at the crossover transition. Calculations in the Quark Model (QM) [10, 11, 12] as well hadron spectrum studies on the lattice [13] predict many more open charm baryons than experimentally detected and tabulated in the Particle Data Table [14], whereas the charmed meson sector is in good agreement $[15,16]$. For instance excitations which are $700 \mathrm{MeV}$ above the ground state are known for the $D, D_{s}$ mesons. On the other hand, the presence of the additional baryon states and resonances would have an impact on the pressure at the freezeout. In the left panel of Fig. 3 we look at a particular charmed baryon $\Lambda_{c}$. Only three states above the ground state are measured in the experiments with well defined spin and parity and the ground state spin is also not measured with certainty yet. There are many more bound states in different spin-parity channels predicted from the QM. The lattice spectrum calculations yield very similar results. Many of the baryon states which have masses $1 \mathrm{GeV}$ or more than the ground state within the blue band in Fig. 3 may be broad resonances which decay fast or would have negligible contribution to the partial pressures. To have a systematic understanding of the relative contribution of these states we compare with our lattice data, the pressure due to a hadron resonance gas (HRG) with all predicted states in the Quark Model (QM-HRG) [12] denoted by brown lines, a QM-HRG-3 consisting of Quark Model states below $3 \mathrm{GeV}$ mass and a PDG-HRG [14] consisting of all the experimentally known states. We consider the partial pressure of charmed baryons with $C=1$ normalized by the partial pressure of the corresponding meson sector introduced in the earlier section, since ratios are not affected by lattice cut-off effects. Using appropriate susceptibilities, we also study the partial pressures in sectors with specific quantum numbers. In the charged sector, charmed baryon and meson partial pressures are given as $P_{B} \simeq \chi_{112}^{B Q C}$ and $P_{M} \simeq \chi_{13}^{Q C}-\chi_{112}^{B Q C}$ whereas for the charmed baryons with strangeness $P_{B} \simeq \chi_{112}^{B S C}$ and $P_{M} \simeq \chi_{13}^{S C}-\chi_{112}^{B S C}$. The results are shown in the right panel of Fig. 3. In all these sectors we find a significant contribution of the additional baryon states over PDG-HRG at the crossover transition and our lattice data provides support in favour of the existence of these additional states.

\section{Conclusions}

We addressed the issue of melting of hadron states consisting of light and strange quarks or the charm quarks. From our analysis of the correlations between the net baryon number and charm and their fluctuations on the lattice, we are able to disentangle the partial pressures of the meson and baryon sectors with different charm content. The open charm mesons and baryons are shown to melt at the chiral crossover transition irrespective of the details of the hadron spectrum and lattice cut-off effects. We are, for the first time able to extract the partial pressures of 

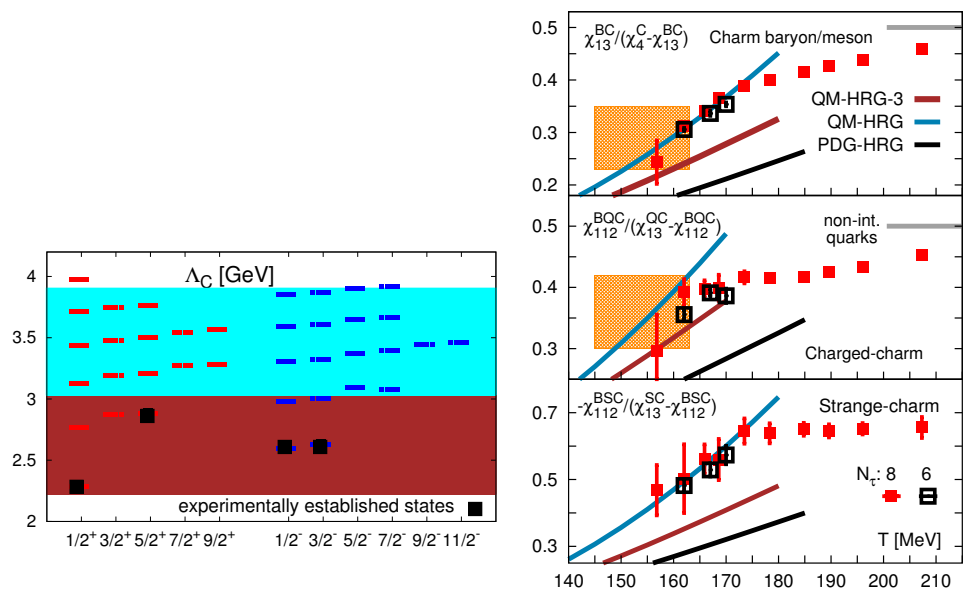

Figure 3. The spectrum of charm Lambda baryons from the Quark Model calculations in the left panel has many more states that are not yet detected in the experiments. The brown band represents excitations of charm Lambda baryon with masses less than $3 \mathrm{GeV}$ and the blue band represents all such known states. In the right panel, our lattice QCD data of the ratio of partial pressures of the open charm mesons and baryons supports the existence of the additional charm baryon states in different channels which are predicted from the Quark Model and hadron spectrum studies on the lattice.

charmed baryon channel and even study the charmed baryons with different charge and strangeness content in QCD. This gives us a tool to understand the details of the charmed hadron spectrum in the crossover transition region. Quark Model as well as hadron spectrum studies on the lattice indicate the presence of many more charmed baryon states than those which have been measured in the experiments. These states would contribute to the thermodynamic quantities near the freezeout. Our data provide hints in support of the existence of these additional baryon states. These additional baryons would contribute to the hadronization of the quark gluon plasma believed to be formed in the heavy ion collision experiments and influence the abundances of hadrons through feed down corrections. Such hadrons consisting of heavy and light quark flavours also play a role in the dissociation of charmonium states and these additional states should be taken into account in all such considerations. A similar analysis has been recently performed for the strange sector as well [17] which also leads to evidence for the presence of additional strange baryons and has important consequences like a lower strangeness freezeout temperature.

\section{Acknowledgements}

This work has been supported in part through contract DE-AC02-98CH10886 with the U.S. Department of Energy, through Scientific Discovery through Advanced Computing (SciDAC) program funded by U.S. Department of Energy, Office of Science, Advanced Scientific Computing Research and Nuclear Physics, the BMBF under grant 05P12PBCTA, EU under grant 283286 and the GSI BILAER grant. Numerical calculations have been performed using GPU-clusters at JLab, Bielefeld University, Paderborn University, and Indiana University. We acknowledge the support of Nvidia through the CUDA research center at Bielefeld University.

\section{References}

[1] H. T. Ding et al., Phys. Rev. D 86 (2012) 014509 [arXiv:1204.4945 [hep-lat]].

[2] P. Petreczky, C. Miao and A. Mocsy, Nucl. Phys. A 855 (2011) 125 [arXiv:1012.4433 [hep-ph]].

[3] T. Matsui and H. Satz, Phys. Lett. B 178 (1986) 416.

[4] A. Bazavov et al., Phys. Rev. Lett. 111 (2013) 082301 [arXiv:1304.7220 [hep-lat]].

[5] R. Bellwied, S. Borsanyi, Z. Fodor, S. D. Katz and C. Ratti, Phys. Rev. Lett. 111 (2013) 202302 [arXiv:1305.6297 [hep-lat]].

[6] J. Stachel, A. Andronic, P. Braun-Munzinger and K. Redlich, J. Phys. Conf. Ser. 509 (2014) 012019 [arXiv:1311.4662 [nucl-th]].

[7] Y. Maezawa, A. Bazavov, F. Karsch, P. Petreczky and S. Mukherjee, PoS LATTICE2013 (2013) 149 [arXiv:1312.4375 [hep-lat]].

[8] A. Bazavov et al. [HotQCD Collaboration], Phys. Rev. D 85 (2012) 054503 [arXiv:1111.1710 [hep-lat]].

[9] A. Bazavov et al., Phys. Lett. B 737 (2014) 210 [arXiv: 1404.4043 [hep-lat]]. 
[10] S. Capstick and N. Isgur, Phys. Rev. D 34 (1986) 2809.

[11] V. Crede and W. Roberts, Rept. Prog. Phys. 76 (2013) 076301 [arXiv:1302.7299 [nucl-ex]].

[12] D. Ebert, R. N. Faustov and V. O. Galkin, Phys. Rev. D 84 (2011) 014025 [arXiv:1105.0583 [hep-ph]].

[13] M. Padmanath, R. G. Edwards, N. Mathur and M. Peardon, arXiv:1311.4806 [hep-lat].

[14] J. Beringer et al. [Particle Data Group], Phys. Rev. D 86 (2012) 010001.

[15] D. Ebert, R. N. Faustov and V. O. Galkin, Eur. Phys. J. C 66 (2010) 197 [arXiv:0910.5612 [hep-ph]].

[16] G. Moir, M. Peardon, S. M. Ryan, C. E. Thomas and L. Liu, JHEP 1305 (2013) 021 [arXiv:1301.7670 [hep-ph]].

[17] A. Bazavov et al., Phys. Rev. Lett. 113 (2014) 072001 [arXiv: 1404.6511 [hep-lat]]; C. Schmidt, Proceedings of this Conference. 